rührung stehenden Metalltheile aus Gusseisen bestehen, dessen Ausdehnung der des Mauerwerks sehr erheblich näher steht, als die des früher meist verwandten Messings, sind gewiss keine Verschlechterungen.

Dr. Nyrén scheint sich besonders an dem möglichen Temperatureinfluss auf die Eisenmasse vom Pfeiler bis zum Zapfenlager zu stossen; denn seine Bemerkung, dass in München sich die Mikroskope, der Schwere folgend, mehr und mehr setzen, kann doch im Ernst einen Zweifel an der genügenden Festigkeit der Pfeilerköpfe nicht ausdrücken sollen. Es ist aber die ungleiche Einwirkung der Temperatur auf die Pfeilerköpfe, also auch die Lager, durch Holzschutzkasten möglichst vermieden (Küstner hat solche nicht einmal für nöthig gehalten, vergl. Veröffentl. IV der Sternwarte Bonn), und die grosse Masse der Pfeilerköpfe lässt eine gute Wärmeausgleichung erwarten.

Ist somit eine Veränderlichkeit in den Lagern an sich wenig wahrscheinlich, so erscheint es noch um so weniger begründet, sie hier zu suchen, als Dr. Nyrén selbst die Möglichkeit anderer Ursachen anführt. Und in der That ist sein Verdacht gegen die Pfeiler nur zu wohl berechtigt. Dass es ein Fehler war, wenn die Baumeister die Fundamente des Meridiankreises und der Collimatoren mit einander in Verbindung brachten und sie mit Höhlungen versahen, darf wohl nicht ohne Weiteres angenommen werden; die Ausdehnung des Grundgemäuers kann nie zu gross sein, wenn es nur den Umfassungsmauern fern genug bleibt, und die Temperatur wird solche Massen trotz einiger Höhlungen nur sehr langsam beeinflussen. Aber es hat sich bei der Aufstellung gezeigt, dass die Ziegelsteine der durch den Fussboden tretenden Instrumentpfeiler so locker und durch den Mörtel so schlecht verbunden waren, dass sie sich beim vorsichtigen Einhauen der Löcher für die Befestigungsschrauben lösten. Es wurde deshalb unsererseits verlangt, es sollten die ganzen Pfeiler erneuert werden. Winnecke konnte das nicht kurzer Hand veranlassen; er versprach aber unsern Protest zu Protocoll zu nehmen, und vermuthlich werden sich unter den Papieren der Sternwarte noch entsprechende Aufzeichnungen vorfinden. Man hat demnach allen Grund, die Veränderlichkeit, die Prof. Becker übrigens als nicht ungewöhnlich gross bezeichnet, zunächst in den Pfeilern zu suchen.

Auch der Nachtheil, den Dr. Nyrén in der Reduction des Kreisdurchmessers sieht, ist nicht erwiesen. Die Theilfehler des Originalkreises und die Einstellfehler beim Theilen sind von dem Durchmesser des zu theilenden Kreises völlig unabhängig, und da bei einem gut eingestellten Reisserwerk ein Fehler in der Uebertragung kaum anzunehmen ist, so darf man, wenn es sich um keinen grösseren Unterschied des Durchmessers als von drei zu zwei Fuss handelt, die Güte der weiteren und der engeren Theilung sehr nahe gleich annehmen. Die Ausgleichung der Temperatur muss aber bei den neuen, kleineren Kreisen, die viel kräftiger geformt sind, als die grösseren älteren, eine vollkommenere und, in Folge der gegentiberstehenden Metallmassen der Pfeilerköpfe, eine schnellere sein.

Was endlich die Mikroskope betrifft, so schätzt Dr. Nyrén die Sicherheit ihrer Lage nach dem Verhältniss des Abstandes der Theilung von der ersten Haltung zu dem gegenseitigen Abstande der beiden Haltungsringe; in Strassburg etwa ${ }_{5} 5 \mathrm{zu}$ $42 \mathrm{~cm}$. Am Meridiankreis Pulkowa sind die entsprechenden Maasse 7.5 und $6 \mathrm{~cm}$, also wesentlich ungünstiger. Aber es ist uberdies zu beachten, dass hier beide Haltungsringe an demselben Kopf eines zusammengeschraubten Messingröhrenkreuzes sitzen, dass also jedes Mikroskop ganz von der etwaigen. Verdrehung seines Rohrkopfes abhängig ist; und diese ist ohne $Z$ weifel so viel mehr zu befürchten, als eine Versetzung der beiden in $42 \mathrm{~cm}$ Abstand liegenden und e in Gussstück bildenden Lagerringe der langen Mikroskope, dass der Unterschied im Abstande der Theilung (für das Röhrenkreuz ist dieser Abstand $10.5 \mathrm{~cm}$ ) kaum in Betracht kommt. Auch hier erscheint also Dr. Nyrén's Besorgniss als völlig unbegründet. Der grössere Abstand zwischen Theilkreis und Mikroskopträger ist aber absichtlich zu dem Zwecke eingeführt, das Umlegen der Fernrohraxe und besonders das Nivelliren zu erleichtern. Beide Punkte hatten oft Anlass zur Klage gegeben.

Nach alle diesem darf wohl gesagt werden, dass die Proclamation solcher Grundregeln, wie Dr. Nyrén sie S. 234 unten aufstellt, keinerlei Werth hat, wenn sie nicht besser begründet werden können; sie werden sonst nur irreleiten und vernünftige Versuche zu Besserungen zurückhalten. Es ist aber auch unverständlich, wie Dr. Nyrén zu dem Ausspruch kommt, die besprochenen Neuerungen seien »bedenklich«. Denn er hebt selbst hervor, dass die Beobachtungen in Strassburg von ungünstigen Umständen beeinflusst sind; er traut besonders den Pfeilern, oder vielmehr ibren Fundamenten nicht und giebt $z u$, dass die Genauigkeit der Endresultate unter dem mehrfachen Wechsel der Beobachter gelitten hat. Er erklärt aber dennoch diese Resultate von ungefähr derselben Genauigkeit, wie Winnecke sie am Meridiankreise in Pulkowa erreicht hat, also ein ungewöhnlich geschickter Beobachter in einheitlicher Arbeit an einem grösseren Instrument ( $61 / 2$ Fuss Brennweite), das meiner persönlichen Ansicht nach in solchen Händen mit den späteren, stärker gebauten und durch ihre Einrichtungen weniger empfindlichen Meridiankreisen wohl concurriren kann. Dies Urtheil bedeutet doch immerhin einen Fortschritt, der vermuthlich unter günstigen Umständen gesteigert werden wird, und giebt durchaus keinen Anlass, die Neuerungen als bedenklich hinzustellen.

Hamburg, 1900 Dec. 2.

F. Repsold.

\title{
Bemerkungen betreffend photographische Nachsuchungen nach (132) Aethra.
}

Herr A. Charlois hat im Bulletin Astr. die Plattencentra von 1893 und 1896 bekannt gegeben, welche gegen gewisse Elementensysteme von (132) Aethra entscheiden können. Diese Aufnahmen machen es ziemlich unwahrscheinlich, dass die mittlere Bewegung liegt I) zwischen $886^{\prime \prime}$ und $890^{\prime \prime}$, 2) zwischen $892^{\prime \prime}$ und $896^{\prime \prime}, 3$ ) zwischen $902^{\prime \prime}$ und $918^{\prime \prime}$, 4) zwischen $923^{\prime \prime}$ und $926^{\prime \prime}$ und 5) zwischen 927" und 934". Es wäre wünschenswerth, wenn auch die übrigen photo. 
graphirenden Astronomen, z. B. Herr Coddington, Datum, RA. und Decl. der Plattencentra, sowie Dimensionen der Platten in $\alpha$ und $\delta$ bekannt geben würden. Ich erlaube mir hervorzuheben, dass der Knoten der Aethra sicher bestimmt ist, und dass es sich daher und mit Rücksicht auf die Lage des Perihels besonders empfiehlt, in der Gegend des absteigenden Knotens RA. $=5^{\mathrm{h}} 2 \mathrm{O}^{\mathrm{m}}$ ein möglichst grosses Gebiet häufig photographisch aufzunehmen. Vor dem absteigenden
Knoten bewegt sich Aethra im Bereiche der photographischen Zonen von Oxford und namentlich längere Zeit in dem von Potsdam.

Die von mir in A. N. 3253 bezeichnete Gegend des Himmels auch weiterhin photographisch zu überwachen kann ich nur empfeblen, da manches dafür spricht, dass die mittlere tägliche Bewegung nicht sehr viel kleiner als Watson's erster Werth 980" sein werde.

Düsseldorf, 1900 Sept. 6.

Wilhelm Luther.

\section{Nachtrag zu „L' orbita di (433) Eros etc." in A. N. 3678.")}

Affinchè gli astronomi possano dall' effemeride di (433) Eros dedurre esattamente il valore $\Delta a: \Delta t$ per una frazione di gionno do le seguenti correzioni all' effemeride in A. N. 3660,3661 e 3662 . Esse sono dedotte per interpolazione sopra calcoli diretti di 15 in 15 di.

\begin{tabular}{|c|c|c|c|c|c|c|c|c|c|c|c|c|c|c|}
\hline \multicolumn{2}{|c|}{$\begin{array}{l}\text { I }^{\mathrm{h}} \text { Berl. } \\
\text { Igoo }\end{array}$} & $\Delta \alpha$ & & \multicolumn{2}{|c|}{$\begin{array}{l}12^{\mathrm{h}} \text { Berl. } \\
1900\end{array}$} & $\Delta \alpha$ & & \multicolumn{2}{|c|}{$\begin{array}{l}\text { 12 } 2^{\mathrm{h}} \text { Berl. } \\
\text { 1900-OI }\end{array}$} & $\Delta \alpha$ & & \multirow{2}{*}{$\begin{array}{l}\text { I } 2^{\mathrm{h}} \text { Berl, } \\
\text { 190I } \\
\text { Gen. } 29\end{array}$} & \multicolumn{2}{|l|}{$\Delta a$} \\
\hline Sett. & I & -0.53 & $\operatorname{ec} \delta$ & Ott. & 21 & -0.89 & $\operatorname{ec} \delta$ & Dic. & 10 & $-\mathrm{I}^{5} .07$ & $\operatorname{ec} \delta$ & & $-0.38 \mathrm{~s}$ & ec $\delta$ \\
\hline & 6 & -0.57 & $»$ & & 26 & $-0.9^{2}$ & $»$ & & 15 & -1.05 & $\gg$ & Febb. 3 & -0.23 & $\gg$ \\
\hline & II & $-0.6 \mathrm{I}$ & 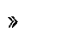 & & 31 & -0.95 & $»$ & & 20 & -1.03 & $\triangleright$ & 8 & -0.09 & $\gg$ \\
\hline & 16 & -0.65 & $\gg$ & Nov. & 5 & -0.98 & 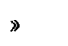 & & 25 & -0.99 & 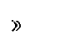 & I 3 & +0.04 & \# \\
\hline & 2 I & -0.69 & $\nabla$ & & 10 & -1.01 & $\gg$ & & 30 & -0.95 & 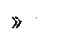 & 18 & +0.16 & » \\
\hline & 26 & -0.73 & $\gg$ & & 15 & -1.04 & $»$ & Gen & 4 & -0.89 & $\gg$ & 23 & +0.28 & $\gg$ \\
\hline Ott. & $\mathbf{I}$ & -0.76 & $»$ & & 20 & -1.06 & $»$ & & 9 & $-0.8 \mathrm{I}$ & $\triangleright$ & 28 & +0.39 & 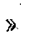 \\
\hline & 6 & -0.79 & » & & 25 & -1.08 & » & & 14 & -0.72 & $»$ & Marz. 5 & $(+0.48)$ & 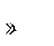 \\
\hline & 11 & -083 & $\gg$ & & 30 & -1.09 & $»$ & & 19 & -062 & $\gg$ & & & \\
\hline & 16 & -0.86 & $\Rightarrow$ & Dic. & 5 & -1.08 & $»$ & & 24 & -0.51 & $»$ & & & \\
\hline
\end{tabular}

In quanto alle correzioni $\Delta \delta$ esse sono minime, e crescono soltanto da gennaio in poi.

\begin{tabular}{|c|c|c|c|c|c|}
\hline I 901 & $\Delta \delta$ & 1901 & $A \delta$ & 1901 & $\Delta \delta$ \\
\hline Gen. 4 & $-3 . \mathrm{I}$ & Gen. 29 & - 6.8 & Febb.23 & $-15 ! 3$ \\
\hline 9 & -3.2 & Febb. 3 & -8.6 & 28 & -16.8 \\
\hline 14 & -3.5 & 8 & -10.4 & Marz. 5 & $(-18.1)$ \\
\hline 19 & -4.0 & 13 & -12.1 & & \\
\hline 24 & -5.2 & 18 & -13.7 & & \\
\hline
\end{tabular}

I nuovi elementi, da cui derivano le prefate correzioni, sono mantenuti osculanti al 3 I ottobre I900.

Roma, 1900 Dic. 19.

E. Millosevich.

*) Aus der Beilage zu Nr. 3678 wiederholt.

\section{Berichtigung zu den BD.-Karten.}

Herr Dr. Hartwig macht mich darauf aufmerksam, dass die drei Sterne

$$
\begin{aligned}
& \text { BD. }+53^{\circ} \cdot 420 \quad 8^{\mathrm{m}} \cdot 5 \mathrm{I}^{\mathrm{h}} 4^{6^{\mathrm{m}} \mathrm{I}} 7^{\mathrm{s}} \cdot 5+53^{\circ} 3^{6: 9} \\
& \text { BD. + } 53.42 \text { I } \quad 9.5 \quad \text { I } 46 \quad 38.4 \quad+53 \quad 18.7 \\
& \begin{array}{lllllll}
\mathrm{BD} .+53.422 & 9.4 & \text { I } & 46 & 40.5 & +53 & 26.4
\end{array}
\end{aligned}
$$

auf Karte 25 nicht ganz richtig eingezeichnet seien. Ich finde, dass thatsächlich die RA. derselben um $30^{5}$ gleich $1.5 \mathrm{~mm}$ zu klein gemacht ist. Der Fehler ist in beiden Auflagen zu berichtigen.

Bonn, 1900 Nov. 22.

F. Küstner.

\section{Beobachtung kleiner Planeten.}

(237) Coolestina. (447) [1899 ES]. 190 I Jan. $98^{\mathrm{h}} 48^{\mathrm{m}} \cdot{ }_{2}$ M. Z. Königstuhl.

RA. $=121^{\circ} 52^{\prime}$ PD. $=62^{\circ} 15^{\prime}$ tgl. Bew. $-13^{\prime}-9^{\prime}$ Gr. 12.0 RA. $=12531$ PD. $=6520$ tgl. Bew. - Ir -4 Gr. i 2.0.

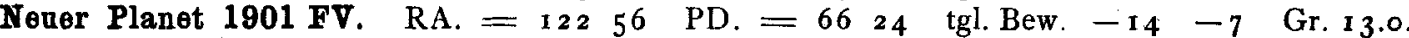

